\title{
Efeito de Glomus etunicatum e fósforo no crescimento inicial de espécies arbóreas em semeadura direta ${ }^{(1)}$
}

\author{
Waldo Wilfredo Flores-Aylas ${ }^{(2)}$, Orivaldo José Saggin-Júnior ${ }^{(3)}$, José Oswaldo Siqueira(4) e Antônio Cláudio Davide ${ }^{(5)}$
}

Resumo - O objetivo deste trabalho foi avaliar os efeitos da disponibilidade de P no solo, da micorriza formada por Glomus etunicatum e de Mycoform, um estimulante desta última, no crescimento e competição inicial de seis espécies arbóreas semeadas diretamente. $\mathrm{O}$ trabalho foi realizado em casa de vegetação com as espécies Senna macranthera (fedegoso), Guazuma ulmifolia (mutamba), Senna multijuga (cássia-verrugosa), Solanum granuloso-leprosum (gravitinga), Schinus terebenthifolius (aroeira) e Trema micrantha (trema), em solo com níveis de P na solução considerados muito baixo, baixo e alto, com inoculação ou não do fungo micorrízico arbuscular G. etunicatum, além do tratamento G. etunicatum + Mycoform. O crescimento das mudas respondeu à inoculação em $\mathrm{P}$ muito baixo e baixo. As mudas apresentaram moderada dependência das micorrizas, não respondendo ao G. etunicatum em P alto. Gravitinga morreu em P muito baixo, mas foi dominante com P baixo e alto. Fedegoso foi dominante com P muito baixo, mostrando-se adaptado à baixa fertilidade. G. etunicatum influenciou a dominância das espécies, auxiliando as menos competitivas e gerando maior equilíbrio. Mycoform influenciou pouco o crescimento, nutrição e competição. O crescimento de espécies pioneiras semeadas diretamente é favorecido pela elevação do $\mathrm{P}$ e pelas micorrizas, as quais também favorecem o equilíbrio entre espécies.

Termos para indexação: fungo micorrízico, inoculação, reflorestamento, floresta tropical, adubação.

\section{Effects of Glomus etunicatum and phosphorus on initial growth of woody species at direct seeding}

Abstract - The objective of this work was to evaluate the effects of soil P availability, mycorrhiza and a mycorrhiza stimulatory product (Mycoform) upon growth and initial competition of six sown woody species, in the greenhouse. The species Senna macranthera, Guazuma ulmifolia, Senna multijuga, Solanum granuloso-leprosum, Schinus terebenthifolius and Trema micrantha were sown together in a soil with very low, low and high levels of $P$ in soil solution combined with inoculation treatments of the arbuscular mycorrhizal fungus Glomus etunicatum, G. etunicatum + Mycoform and a non-inoculated control. Growth response to mycorrhizal inoculation occurred with very low and low phosphorus. The seedlings presented moderate mycorrhizal dependency, because there was no response with high phosphorus. Solanum granuloso-leprosum died with very low P, but was the dominant specie with low and high P, and very responsive to phosphorus. Senna macranthera was dominant only with very low P, indicating that it is quite adapted to low fertility soils. Glomus etunicatum influenced the species dominance, promoting the least competitive species and favoring uniformity in plant growth. Growth, nutrition and competition were little influenced by Mycoform. The growth of pioneer species is favored by increasing $\mathrm{P}$ availability and by mycorrhiza which also favores the growth equilibrium between them in mixed and direct seeding on low fertility soil.

Index terms: mycorrhizal fungi, inoculation, reforestation, tropical forests, fertilizing.

(1) Aceito para publicação em 11 de novembro de 2002. Trabalho financiado pelo CNPq.

(2) Universidade Federal de Lavras (Ufla), Caixa Postal 37, CEP 37200-000 Lavras, MG. Bolsista da Capes. E-mail: waldoflores@zipmail.com.br

(3) Embrapa-Centro Nacional de Pesquisa de Agrobiologia, Caixa Postal 74505, CEP 23851-970 Seropédica, RJ. E-mail: saggin@cnpab.embrapa.br

(4) Ufla, Dep. de Ciência do Solo. Bolsista do CNPq. E-mail: siqueira@ufla.br

(5) Ufla, Dep. de Ciências Florestais. Bolsista do CNPq. E-mail: acdavide@ufla.br

\section{Introdução}

Cerca de 180 milhões de hectares de florestas foram destruídas no mundo a partir da década de 80 (FAO, 1997). Além do impacto ambiental, a destruição deste importante recurso natural tem efeito acentuado na qualidade de vida e na atividade sócio-econômica, trazendo prejuízos à ecologia das regiões sob forte interferência do desmatamento e representando séria ameaça ao futuro da humanidade. A necessidade de recuperar essas áreas degra- 
dadas exige procedimentos técnicos e economicamente viáveis, como o emprego de espécies arbóreas nativas de crescimento rápido (pioneiras), de grande potencial para tal finalidade por apresentarem alta capacidade de absorver nutrientes, rápido crescimento e imobilização de carbono e nutrientes na fitomassa, evitando a sua transferência para fora do sistema (Montagnini \& Sancho, 1994) e facilitando o reestabelecimento da ciclagem de $\mathrm{C}$ e nutrientes no sistema solo-planta.

No Brasil, a revegetação com espécies arbóreas nativas é pequena comparada ao uso de espécies exóticas de interesse econômico, como o eucalipto, em parte por causa da falta de conhecimento sobre as espécies nativas em plantios artificiais, falta de tecnologia na implantação de áreas extensas e custo das mudas. As espécies pioneiras de crescimento rápido são geralmente muito responsivas à adubação mineral (Carneiro et al., 1996; Lima et al., 1997), particularmente ao $\mathrm{P}$, que na maioria dos solos tropicais e, principalmente, naqueles degradados, é um dos nutrientes mais limitantes do crescimento e estabelecimento de plantas (Siqueira \& Saggin Júnior, 1995). Assim, a simbiose com os fungos micorrízicos arbusculares (FMAs) torna-se importante na revegetação de áreas degradadas com espécies nativas, por contribuir para o melhor aproveitamento desse nutriente no solo (Souza \& Silva, 1996).

A inoculação de FMAs pode ser praticada na formação de mudas (Saggin Júnior \& Lovato, 1999), garantindo o estabelecimento da simbiose. Em semeadura direta, a inoculação torna-se mais difícil por causa da necessidade de grande quantidade de inoculante. Neste caso, a aplicação de substâncias estimulantes da micorrização (Siqueira et al., 1991) pode favorecer a colonização micorrízica das raízes a partir de propágulos existentes no solo (Silva Júnior \& Siqueira, 1998).

Os FMAs promovem crescimento diferenciado entre as espécies nativas, com forte interação com os níveis de P no solo (Siqueira \& Saggin Júnior, 2001). Este efeito pode influenciar o crescimento inicial (Siqueira et al., 1998) e, possivelmente, também a sua capacidade de sobrevivência e competição (Brandon et al., 1997) em plantios mistos, como em semeadura direta. Janos (1987) propõe que a sucessão e a diversidade de espécies vegetais em flores- tas tropicais sejam influenciadas pela interconexão entre plantas através da rede de hifas de fungos micorrízicos e pela dependência micorrízica que as espécies apresentam.

A semeadura direta de espécies florestais é um sistema de regeneração alternativo já testado no exterior para reduzir custos com fertilizantes e mão-de-obra. Na Austrália, é uma opção viável para Acacia spp. e Eucalyptus spp., em áreas com baixa precipitação e solos arenosos (Knight et al., 1998). No Brasil, existem estudos com Pinus taeda L., que indicam o outono como época ideal de semeadura (Mattei, 1993), e com espécies pioneiras nativas, que indicam baixo custo e viabilidade da semeadura aérea de manacás-da-serra (Tibouchina spp.) e jacatirões (Miconia spp.) em clareiras da Serra do Mar, em Cubatão, SP, a partir de sementes peletizadas com gel hidrofílico (Pompéia et al., 1989). No entanto, nem a influência do P nem da micorrização foi estudada em relação ao comportamento de plantas arbóreas nativas em semeadura direta no Brasil.

O objetivo deste trabalho foi avaliar os efeitos da disponibilidade de $\mathrm{P}$ no solo, da micorriza formada por Glomus etunicatum e de Mycoform, um estimulante desta última, no crescimento e competição inicial de seis espécies arbóreas semeadas diretamente.

\section{Material e Métodos}

O trabalho foi realizado em casa de vegetação do Departamento de Ciência do Solo (DCS) da Universidade Federal de Lavras (Ufla), MG, entre dezembro de 1998 e abril de 1999, num Latossolo Vermelho-Escuro distrófico, coletado na camada de 0-50 cm, no Distrito de Jaguara, Município de São João del Rei, MG, com vegetação original de mata subcaducifólia e atualmente utilizada como pastagem nativa. O material do solo foi homogeneizado, secado e peneirado em malhas de $4 \mathrm{~mm}$ e sua análise química apresentou pH em água (1:2,5), 5,4; $\mathrm{P} \mathrm{e} \mathrm{K}{ }^{+}$(Mehlich I/ colorimetria e fotometria de chama, respectivamente), $1 \mathrm{e}$ $45 \mathrm{mg} \mathrm{dm}^{-3} ; \mathrm{Ca}^{2+}, \mathrm{Mg}^{2+} \mathrm{e}^{3+}\left(\mathrm{KCl} 1 \mathrm{~mol} \mathrm{~L}^{-1} /\right.$ titulometria) ), respectivamente, $0,7,0,5$ e $0,2 \mathrm{mg} \mathrm{dm}^{-3} ; \mathrm{S}$ (fosfato de cálcio dihidratado/turbidimetria), $5,5 \mathrm{mg} \mathrm{dm}^{-3} ; \mathrm{H}^{+}+\mathrm{Al}^{3+}$ $\left(\mathrm{CaCl}_{2}\right.$ 0,01 $\mathrm{mol} \mathrm{L}^{-1} /$ peagometria), $6,3 \mathrm{mg} \mathrm{dm}^{-3}$; matéria orgânica (dicromato de sódio e ácido sulfúrico/titulometria), $29 \mathrm{~g} \mathrm{~kg}^{-1} ; \mathrm{V} \%$ (calculado), 17. Aplicou-se 1,5 g de calcário dolomítico por kg de solo. Em seguida, essa mistura foi umedecida e incubada por 15 dias, quando apresentou $\mathrm{pH}, 6,2$ e V\%, 55. Depois o solo foi secado e 
fumigado com $263 \mathrm{~cm}^{3} \mathrm{~m}^{-3}$ de Bromex (Brometo de metila $98 \%$ + cloropicrina $2 \%$ ), arejado por quatro dias e adubado com $100 \mathrm{mg} \mathrm{kg}^{-1}$ de $\mathrm{KCl}$, antes de ser colocado em bandejas de polietileno com capacidade para $48 \mathrm{~kg}$, empregadas como unidades experimentais. Em cada bandeja foram semeadas seis espécies arbóreas em sulcos espaçados de $8 \mathrm{~cm}$ (uma espécie por sulco). Aos 30 dias após a semeadura foi realizado desbaste, mantendo-se sete plantas por espécie, espaçadas entre si por $5 \mathrm{~cm}$.

As sementes, fornecidas pelo Laboratório de Sementes Florestais da Ufla, foram armazenadas a $5^{\circ} \mathrm{C}$ e $75 \%$ de umidade relativa até serem semeadas. As espécies estudadas foram fedegoso (Senna macranthera (Collad) Irwin et Barneby), mutamba (Guazuma ulmifolia Lamark), cássia-verrugosa (Senna multijuga (Rich.) Irwin et Barneby), aroeira (Schinus terebenthifolius Raddi), gravitinga (Solannum granuloso-leprosum Dunal) e trema (Trema micrantha (L.) Blume), as quais receberam combinações de três tratamentos de inoculação de FMA e três níveis de P na solução do solo, em esquema fatorial 3x3, seguindo um delineamento experimental inteiramente casualizado, com quatro repetições. Os tratamentos foram: inoculação de Glomus etunicatum (GE), inoculação de Glomus etunicatum e aplicação de Mycoform (GE+Mycoform), e controle sem inoculação (SI), combinados com os níveis de $\mathrm{P}$ na solução do solo $\left(\mathrm{mg} \mathrm{L}^{-1}\right)$ de 0,002 (muito baixo), 0,02 (baixo) e 0,2 (alto), definidos como deficiente, ideal para a micorrização e ótimo para o crescimento da maioria das espécies vegetais, respectivamente (Habte \& Manjunath, 1991). A concentração de $0,002 \mathrm{mg} \mathrm{L}^{-1}$ de $\mathrm{P}$ era natural do solo utilizado e as doses necessárias de $\mathrm{P}$ para atingir as concentrações $0,02 \mathrm{e}$ $0,2 \mathrm{mg} \mathrm{L}^{-1}$ foram 52 e $682 \mathrm{mg} \mathrm{kg}^{-1}$ de P no solo, respectivamente, aplicadas na forma de superfosfato triplo $(0,3 \mathrm{e}$ $\left.3,8 \mathrm{~g} \mathrm{~kg}^{-1}\right)$, as quais foram completamente homogeneizadas com o solo uma semana antes da semeadura. As doses de $\mathrm{P}$ para atingir as concentrações almejadas foram estimadas após ensaio de incubação do solo e análise da solução do solo (Manjunath \& Habte, 1990). As concentrações de P disponível no solo nos três níveis de $\mathrm{P}$, extraído por Mehlich I, foram 1, 3 e $119 \mathrm{mg} \mathrm{dm}^{-3}$, respectivamente.

Utilizou-se Glomus etunicatum Becker \& Gerdemann, oriundo de cafezais de Varginha, MG, mantido e multiplicado por vários anos em casa de vegetação com Brachiaria decumbens Stapf como planta hospedeira, por sua eficiência em promover o crescimento de espécies arbóreas nativas (Saggin Júnior, 1997). Sua inoculação foi realizada colocando-se solo-inóculo contendo 20 esporos $\mathrm{mL}^{-1}$, além de hifas e raízes colonizadas no sulco de semeadura. Em cada sulco, colocaram-se $20 \mathrm{~mL}$ de solo-inóculo, totalizando 400 esporos por sulco (2.400 esporos por bandeja). Para diminuir as diferenças da microbiota entre os tratamentos, adicionaram-se, por bandeja, $330 \mathrm{~mL}$ de filtrado do inóculo, isento de propágulos de FMAs.

No fundo de cada sulco, após a aplicação do inóculo, aplicou-se Mycoform, no tratamento pertinente. O Mycoform, cujo ingrediente ativo é a formononetina (4'metoxi 7-hidroxiisoflavona), foi aplicado na dose de $51,25 \mu \mathrm{g} \mathrm{kg}^{-1} \mathrm{de}$ formononetina no solo. Em seguida, colocou-se fina camada de solo, sobre a qual foram depositadas as sementes. A quebra da dormência das sementes de fedegoso, trema, aroeira, gravitinga e cássia-verrugosa foi feita conforme Davide et al. (1995), e na mutamba as sementes permaneceram por 50 minutos em ácido sulfúrico (determinado em pré-teste). Após a quebra da dormência, realizou-se a desinfestação com hipoclorito de sódio $\left(10 \mathrm{~mL} \mathrm{~L}^{-1}\right)$ e a semeadura direta de 35 sementes de cada espécie por sulco, as quais foram cobertas com outra fina camada de solo. Até os 20 dias após a semeadura (DAS), as bandejas foram mantidas sob tela sombrite com $50 \%$ de sombreamento. Após 70 DAS, aplicou-se solução de Hoagland (completa exceto P) $50 \%$ de força. Essa operação foi repetida aos 80 e $90 \mathrm{DAS}$, porém também sem potássio. Ao longo do período experimental a radiação incidida na casa de vegetação foi em média $1.500 \mu \mathrm{mol} \mathrm{m}^{-2} \mathrm{~s}^{-1}$, medida com aparelho portátil IRGA/ ADC-LCA 4. No controle de pulgão, em plantas de gravitinga, aos 100 DAS pulverizou-se Confidor Grda $0,25 \mathrm{~g} \mathrm{~L}^{-1}$. A umidade do solo foi mantida entre $60 \% \mathrm{e}$ $70 \%$ do volume total de poros por irrigações periódicas com água filtrada deionizada.

Aos 60 DAS, avaliou-se a altura das sete plantas de cada espécie e três delas foram coletadas alternadamente nas bandejas, mantendo-se as quatro plantas restantes de cada espécie. Nas plantas coletadas avaliaram-se a área foliar, a massa da parte aérea seca e a colonização micorrízica. A área foliar foi determinada com medidor portátil modelo LICOR CI 3000A. A massa da parte aérea seca foi medida após sua lavagem em água de torneira, seguida de lavagem em água destilada e secada em estufa com circulação de ar a $68^{\circ} \mathrm{C}$. A parte aérea foi moída e os teores dos nutrientes nos tecidos foram determinados, após a extração destes por digestão nítrico-perclórica. O P por colorimetria, o K por fotometria de chama, o $\mathrm{S}$ por turbidimetria e o $\mathrm{Ca}, \mathrm{Mg}$, $\mathrm{Cu}, \mathrm{Zn}, \mathrm{Mn}$ e Fe por espectrofotometria de absorção atômica. $\mathrm{O} \mathrm{N}$ foi determinado pelo método de Kjeldahl modificado após digestão sulfúrica e o $\mathrm{B}$ foi extraído pelo método de água quente. Das raízes frescas retiraram-se amostras de $1 \mathrm{~g}$ de raízes finas para avaliação da colonização micorrízica pelo método da placa quadriculada (Giovannetti \& Mosse, 1980), após clarificação com KOH $\left(10 \mathrm{~mL} \mathrm{~L}^{-1}\right)$ e coloração com azul tripano (Phillips \& 
Hayman, 1970). Aos 120 DAS, coletaram-se as quatro plantas restantes que foram avaliadas tal qual as coletadas aos 60 DAS. Desta vez, as raízes foram retiradas intactas do solo e separadas por espécies, o que permitiu melhor amostragem.

Os dados de crescimento vegetativo (área foliar e massa da parte aérea seca) foram analisados e expressos pelo total da bandeja (somatório de todas as espécies) e pela produção de cada espécie individualmente. Os dados obtidos foram submetidos à análise de variância e as médias comparadas pelo teste de Tukey a 5\% utilizando-se o programa estatístico SAEG (Universidade Federal de Viçosa). Na obtenção da homocedastidade, os dados de colonização micorrízica foram transformados segundo a expressão $\log (\mathrm{x}+2,5)$.

\section{Resultados e Discussão}

Os efeitos dos tratamentos de inoculação na produção de massa da parte aérea seca nas bandejas foram evidentes aos 60 DAS e ampliados aos 120 DAS (Figura 1). Os efeitos da inoculação de GE foram observados nos níveis 0,002 e $0,02 \mathrm{mg} \mathrm{L}^{-1} \mathrm{de} \mathrm{P}$ na solução do solo, porém, deixaram de existir no nível 0,2 $\mathrm{mg} \mathrm{L}^{-1}$ de $\mathrm{P}$, considerado ótimo para o desenvolvimento da maioria das espécies vegetais (Habte \& Manjunath, 1991). Em relação ao P naturalmente disponível no solo $\left(0,002 \mathrm{mg} \mathrm{L}^{-1}\right)$, a elevação para $0,2 \mathrm{mg} \mathrm{L}^{-1}$ de $\mathrm{P}$ na solução do solo resultou em aumentos significativos na produção de massa da parte aérea seca em todos os tratamentos de inoculação, nas duas épocas avaliadas. Efeitos semelhantes foram observados em relação à área foliar total nas bandejas, de forma que estes dados não serão apresentados.

A contribuição relativa de cada espécie em relação ao total de massa da parte aérea seca por bandeja foi muito semelhante aos 60 e 120 DAS, por isso são apresentados apenas os dados referentes aos 120 DAS (Figura 2). Os tratamentos de inoculação alteraram a contribuição relativa das espécies quanto ao total de massa produzida, indicando ter alterado a competição entre as espécies, e esse efeito foi altamente influenciado pelo nível de $\mathrm{P}$ no solo. No nível muito baixo de $\mathrm{P}\left(0,002 \mathrm{mg} \mathrm{L}^{-1}\right)$, nas bandejas sem inoculação, houve um crescimento muito maior do fedegoso em relação às outras espécies, e esta espécie foi responsável por mais de $50 \%$ do total de massa seca produzida. A cássia-verrugosa também apresentou crescimento destacado, contribuindo com $24 \%$ do total de massa seca. Nessas condições, a gravitinga e a trema cresceram muito pouco, contribuindo cada uma com $2 \%$ do total de massa seca produzida. Na presença de GE houve aumento na produção de massa seca de todas as espécies, reduzindo a dominância de fedegoso e cássia-verrugosa e favorecendo gravitinga e mutamba. Nesta condição, (P muito baixo) o crescimento da trema e da aroeira foi muito pequeno.

No nível baixo de $\mathrm{P}\left(0,02 \mathrm{mg} \mathrm{L}^{-1}\right)$ houve forte dominância da gravitinga sobre as outras espécies, e sua contribuição foi mais de $50 \%$ do total de massa seca produzida. Na ausência de micorrizas a dominância foi acentuada, produzindo $92 \%$ do total de massa seca, porém, com a inoculação de GE, as outras espécies tiveram sua contribuição relativa em relação ao total de massa seca ampliada, o que

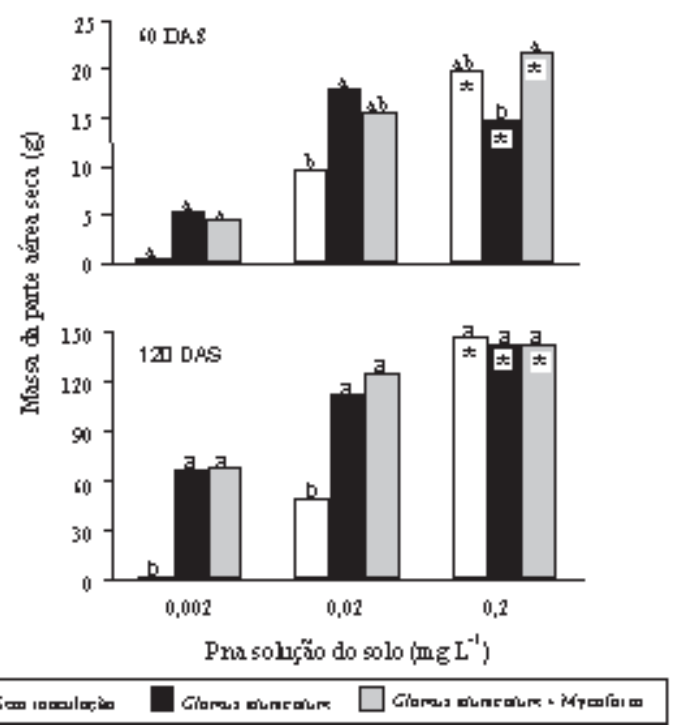

Figura 1. Produção total de massa da parte aérea seca por bandeja das espécies arbóreas germinadas e crescidas com diferentes tratamentos de inoculação de Glomus etunicatum e de níveis de P na solução do solo, em duas épocas de coleta, 60 e 120 DAS. Letras iguais indicam que as médias não possuem diferenças significativas dentro de cada nível de $\mathrm{P}$ e asterisco indica que a média é superior à do nível $0,002 \mathrm{mg} \mathrm{L}^{-1}$, dentro de cada tratamento de inoculação, pelo teste de Tukey a $5 \%$ de probabilidade. 
garantiu crescimento mais equilibrado. No nível alto de $\mathrm{P}\left(0,2 \mathrm{mg} \mathrm{L}^{-1}\right)$, a dominância da gravitinga em relação às outras espécies foi mantida e, na presença de micorrizas, ela contribuiu com cerca de $60 \%$ do total de massa seca; na ausência, com $43 \%$. A contribuição relativa da trema na produção de massa foi sensivelmente ampliada neste nível de $\mathrm{P}$ em todos os tratamentos de inoculação. Houve pouco efeito do Mycoform em alterar a contribuição relativa de cada espécie em relação ao total de massa seca produzida. Apenas plantas de fedegoso responderam significativamente ao Mycoform no nível $0,02 \mathrm{mg} \mathrm{L}^{-1}$ de $\mathrm{P}$, aumentando de $11 \%$ para $19 \%$ sua contribuição no total de massa seca na presença do estimulante.

Nos níveis mais baixos de $\mathrm{P}$, houve efeito positivo da inoculação de GE na altura de todas as espécies arbóreas estudadas (Figura 3). Porém, no nível mais alto de $\mathrm{P}$ não houve este efeito, e na aroeira e na trema o efeito foi negativo. A aplicação de Mycoform
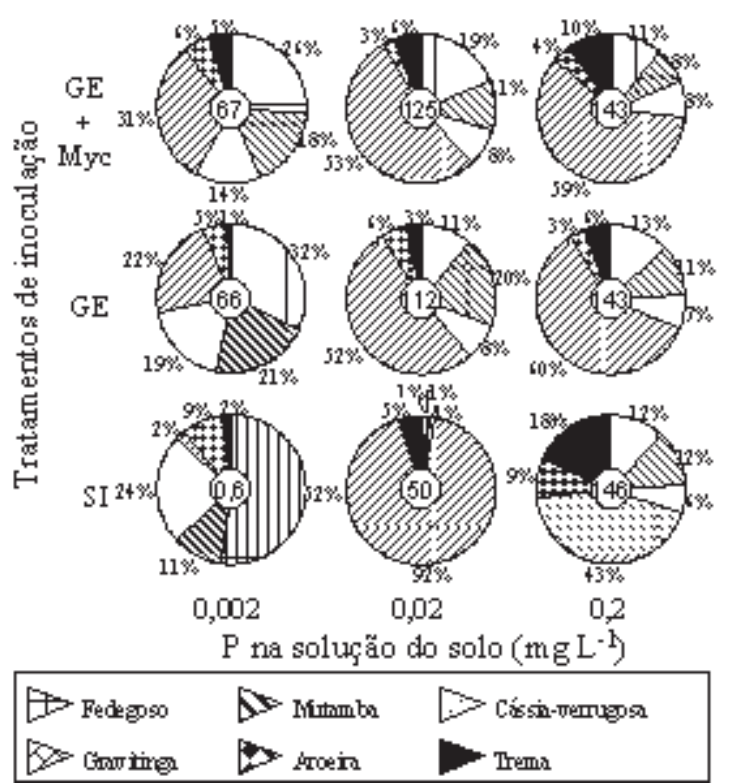

Figura 2. Contribuição relativa de cada espécie arbórea para o total de massa de parte aérea seca (nos círculos centrais em gramas) produzida em bandeja, aos 120 DAS. Os tratamentos foram: sem inoculação (SI), inoculação de Glomus etunicatum (GE) e inoculação de G. etunicatum mais aplicação de Mycoform (GE + Myc), combinadas com três níveis de $\mathrm{P}$ na solução do solo. não apresentou efeito significativo na altura das plantas, exceto pequeno efeito negativo na aroeira no nível $0,02 \mathrm{mg} \mathrm{L}^{-1}$ de $\mathrm{P}$ no solo. Ao contrário, a aplicação de P no solo teve efeito positivo acentuado, particularmente na ausência de inoculação de GE. Todas as espécies que não sofreram inoculação responderam positivamente aos $0,2 \mathrm{mg} \mathrm{L}^{-1}$ de $\mathrm{P}$ no solo em relação aos demais níveis. Sem inoculação, a única espécie que apresentou efeito positivo do $\mathrm{P}$ já no nível $0,02 \mathrm{mg} \mathrm{L}^{-1}$ foi a gravitinga, demonstrando grande capacidade de absorção de $\mathrm{P}$ em solos com baixa disponibilidade deste nutriente, mesmo sem micorrizas. Nos tratamentos com GE, o efeito positivo da aplicação de $\mathrm{P}$ foi menor por causa do grande auxílio do fungo na absorção de $\mathrm{P}$, mesmo em solos com baixa disponibilidade deste nutriente. Fedegoso, mutamba e cássia-verrugosa, com inóculo, não apresentaram resposta positiva à aplicação de $\mathrm{P}$ no solo, ao passo que gravitinga, aroeira e trema, variando um pouco com a presença de Mycoform, apresentaram efeito positivo da aplicação de $0,02 \mathrm{mg} \mathrm{L}^{-1}$ de $\mathrm{P}$, e este efeito foi mantido no nível $0,2 \mathrm{mg} \mathrm{L}^{-1}$ apenas na gravitinga e na trema.

Os teores de nutrientes na parte aérea das seis espécies estudadas foram influenciados pelos tratamentos e por suas interações (Tabela 1). Houve pouco efeito da aplicação de Mycoform nos teores de nutrientes das plantas e, quando existente, não apresentavam tendência definida. $O$ efeito da inoculação de G. etunicatum no nível 0,002 $\mathrm{mg} \mathrm{L}^{-1}$ de P, embora evidente em crescimento, não foi possível ser constatado em vários nutrientes por causa da pouca produção de massa seca nas plantas sem inóculo, o que inviabilizou as análises. Isto ocorreu até o nível $0,02 \mathrm{mg} \mathrm{L}^{-1}$ de P na aroeira, na análise do teor de fósforo. A inoculação aumentou o teor de $\mathrm{P}$ na parte aérea no nível $0,02 \mathrm{mg} \mathrm{L}^{-1}$ em fedegoso, mutamba, cássia-verrugosa e gravitinga. No nível $0,2 \mathrm{mg} \mathrm{L}^{-1}$ de $\mathrm{P}$, apenas fedegoso, gravitinga e cássia-verrugosa tiveram teores mais elevados de $\mathrm{P}$ decorrentes da inoculação.

Os teores de $\mathrm{N}$ foram mais elevados no fedegoso do que nas demais espécies, e geralmente menores nas plantas dos tratamentos que promoveram maior produção de massa seca, possivelmente por um efeito de diluição. No nível $0,02 \mathrm{mg} \mathrm{L}^{-1}$ de $\mathrm{P}$, a inoculação reduziu os teores de $\mathrm{N}$ na gravitinga, aroeira e trema; 
no nível 0,2 $\mathrm{mg} \mathrm{L}^{-1}$ não se verificou alteração. Nessas espécies ocorreu redução dos teores de $\mathrm{N}$ com o aumento dos níveis de $\mathrm{P}$ no solo. Os teores de $\mathrm{Cu}$ foram aumentados pela inoculação na aroeira, fedegoso e gravitinga nos níveis mais baixos de $\mathrm{P}$ no solo. $\mathrm{O}$ incremento da disponibilidade de $\mathrm{P}$ no solo tendeu a reduzir os teores de $\mathrm{Cu}$ em todas as espécies. Os teores de $\mathrm{Mn}$ tenderam a ser reduzidos pela inoculação no nível $0,02 \mathrm{mg} \mathrm{L}^{-1}$ de $\mathrm{P}$, exceto na aroeira, em que foram aumentados. Nos demais níveis de $\mathrm{P}$, os efeitos da inoculação foram poucos consistentes, assim como os efeitos dos diferentes níveis de fósforo.

A colonização aos 60 DAS foi muito baixa e sem influência dos tratamentos, por isso os dados não

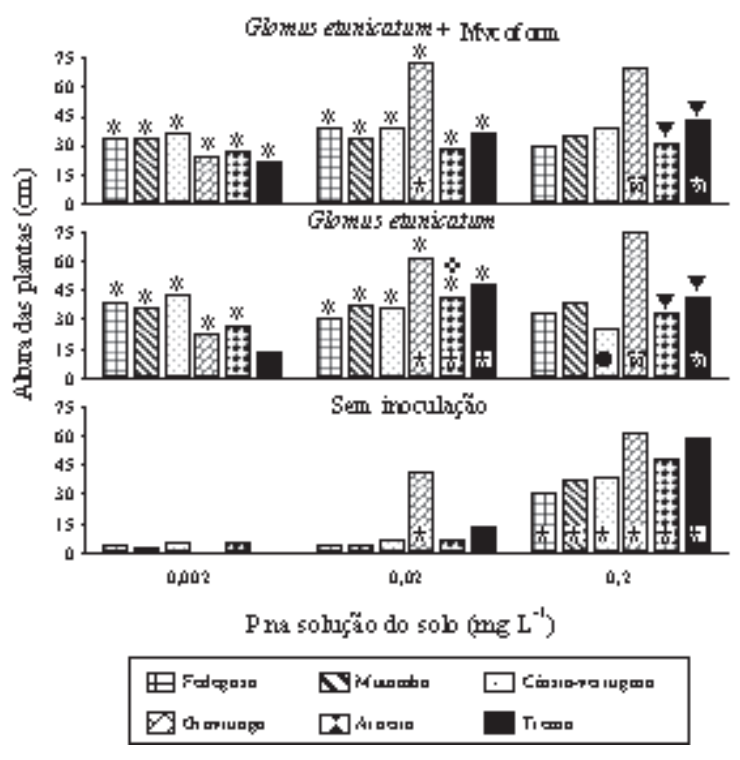

Figura 3. Altura das espécies arbóreas germinadas e crescidas com diferentes tratamentos de inoculação e níveis de P na solução do solo aos 120 DAS. Os símbolos comparam individualmente por espécie os tratamentos de inoculação (símbolos fora das barras) e os níveis de P (símbolos dentro das barras), pelo teste de Tukey a 5\% de probabilidade. *: efeito positivo da inoculação; $\nabla$ : efeito negativo da inoculação; $\$$ : efeito negativo da adição de Mycoform; $\star$ : efeito positivo do nível de $\mathrm{P}$ no nível imediatamente inferior; $\Sigma$ : efeito positivo do nível de $\mathrm{P}$ apenas no nível $0,002 \mathrm{mg} \mathrm{L}^{-1} ; \mathbf{O}$ : efeito negativo do nível de $\mathrm{P}$ nos níveis inferiores. são apresentados, entretanto aos 120 DAS observou-se elevado efeito da inoculação em todas as espécies (Tabela 2). No fedegoso, cássia-verrugosa e aroeira não houve efeito nem dos níveis de $\mathrm{P}$ nem da aplicação de Mycoform sobre a colonização. $\mathrm{Na}$ mutamba e gravitinga, em solo com $0,002 \mathrm{mg} \mathrm{L}^{-1}$ de $\mathrm{P}$, a aplicação de Mycoform aumentou significativamente a colonização. Na gravitinga e trema observou-se efeito contrário com a aplicação de $\mathrm{P}$ ao solo. A elevação na disponibilidade de P no solo reduziu a colonização na mutamba, gravitinga e trema quando foi aplicado Mycoform e não influenciou a colonização das demais espécies.

O crescimento das espécies foi influenciado pela inoculação de GE e pela elevação do P no solo, de acordo com o grau de dependência micorrízica e de resposta ao $\mathrm{P}$ e às micorrizas de cada espécie arbórea. Nos níveis baixos de $\mathrm{P}\left(0,002\right.$ e $\left.0,02 \mathrm{mg} \mathrm{L}^{-1}\right)$ o crescimento e a altura de todas as espécies responderam consistentemente à inoculação de GE (Figura 3). Nesta situação o estresse nutricional foi amenizado pelas micorrizas, como acontece com a maioria das espécies vegetais (Siqueira \& Saggin Júnior, 1995). Isto é evidente ao se observar os teores de nutrientes das plantas quando foi possível analisar os tecidos daquelas que não receberam inóculo no nível $0,002 \mathrm{mg} \mathrm{L}^{-1}$ de fósforo. A micorrização tendeu a aumentar os teores de todos os nutrientes, com exceção dos de nitrogênio. A melhora nutricional promovida pela micorriza foi importante em relação à gravitinga e à trema no nível $0,002 \mathrm{mg} \mathrm{L}^{-1} \mathrm{de} \mathrm{P}$, que praticamente não sobrevivem com tal nível de $\mathrm{P}$ sem micorrizas. O fedegoso mostrou ser a espécie mais adaptada a esta condição extrema de baixa disponibilidade de $\mathrm{P}$, produzindo mais massa que as outras espécies (Figura 2). A gravitinga foi muito responsiva ao $\mathrm{P}$ e beneficiada mesmo na ausência de inoculação, fato também observado na trema, porém em menor magnitude. Desta forma, com 0,02 e 0,2 $\mathrm{mg} \mathrm{L}^{-1} \mathrm{de} \mathrm{P}$ a gravitinga foi a espécie dominante, com $0,2 \mathrm{mg} \mathrm{L}^{-1}$, junto com a trema destacaram-se na produção de massa. Tais resultados corroboram os estudos de Saggin Júnior (1997) de serem estas espécies altamente responsivas ao fósforo.

A resposta da trema e da gravitinga ao $\mathrm{P}$ aplicado diminui a magnitude da resposta destas espécies à micorrização, mesmo em condições em que o supri- 
mento de $\mathrm{P}$ para as plantas seja ótimo somente na presença de micorriza, como em 0,02 $\mathrm{mg} \mathrm{L}^{-1}$ (Habte \& Manjunath, 1991). De acordo com Siqueira \& Saggin Júnior (2001), isto indica que essas espécies, embora responsivas a micorrizas, apresentam baixa dependência micorrízica, deixando de responder à inoculação em condições de maior disponibilidade de $\mathrm{P}$ no solo. A pouca dependência micorrízica da trema, assim como da aroeira, é evidenciada pelo efeito negativo da inoculação no nível $0,2 \mathrm{mg} \mathrm{L}^{-1}$ de $\mathrm{P}$ (Figura 3). Segundo esses autores, quanto menor o nível de $\mathrm{P}$ disponível no solo, no qual as micorrizas deixam de ser benéficas em relação ao crescimento, menor é a dependência micorrízica da planta. Nenhuma das espécies estudadas apresentaram dependência micorrízica extremamente alta, já que não responderam à inoculação com suprimento de $\mathrm{P}$ adequado $\left(0,2 \mathrm{mg} \mathrm{L}^{-1}\right)$. Esse comportamento parece ser uma tendência geral nas espécies pioneiras nativas já estudadas (Carneiro et al., 1996; Paron et al., 1997; Pouyú-Rojas \& Siqueira, 2000). A gravitinga, cássia-verrugosa e fedegoso ainda se beneficiaram das micorrizas na absorção de $\mathrm{P}$, mesmo com alto nível deste nutriente no solo $\left(0,2 \mathrm{mg} \mathrm{L}^{-1}\right)$, tendo-se em vista teores mais elevados de $\mathrm{P}$ na parte aérea (Tabela 1). Porém, tal fato não parece indicar que tenha sido resultante da dependência delas às micorrizas, já que os aumentos nos teores não refletiram em aumentos de crescimento. É possível que essas espécies apresentem consumo de luxo de $\mathrm{P}$ quando são micorrizadas e crescem em solo com teor adequado de P no solo (Smith et al., 1992).

A aplicação de Mycoform influenciou pouco o crescimento e a nutrição das plantas, e os raros efeitos significativos não apresentavam tendência definida. Como o efeito benéfico do Mycoform é o estímulo da colonização inicial (Siqueira et al., 1991), é provável que após 120 dias de crescimento este benefício já tenha ocorrido se consideradas as taxas de colonização observadas. Houve efeito positivo do Mycoform na colonização da mutamba e da gravitinga apenas na ausência de adição de $\mathrm{P}$ ao solo. Já os efeitos inibitórios na colonização da gravitinga e da trema foram observados quando se aplicou $\mathrm{P}$ ao

Tabela 1. Teores de nutrientes na parte aérea de espécies arbóreas nativas 120 DAS, em solo com 0,002, 0,02 e $0,2 \mathrm{mg} \mathrm{L}^{-1}$ de fósforo ${ }^{(1)}$.

\begin{tabular}{|c|c|c|c|c|c|c|c|c|c|c|c|c|}
\hline \multirow{3}{*}{$\begin{array}{l}\text { Tratamento de } \\
\text { inoculação }\end{array}$} & \multicolumn{3}{|c|}{$\mathrm{N}$} & \multicolumn{3}{|c|}{$P$} & \multicolumn{3}{|c|}{$\mathrm{Cu}$} & \multicolumn{3}{|c|}{$\mathrm{Mn}$} \\
\hline & 0,002 & 0,02 & 0,2 & 0,002 & 0,02 & 0,2 & 0,002 & 0,02 & 0,2 & 0,002 & 0,02 & 0,2 \\
\hline & \multicolumn{6}{|c|}{ 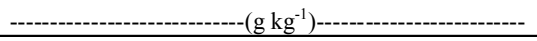 } & \multicolumn{6}{|c|}{ 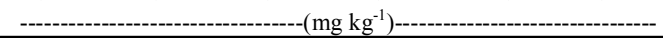 } \\
\hline & & & & & & & Fedegoso & & & & & \\
\hline Sem inoculação (SI) & $61 \mathrm{Aa}$ & $35 \mathrm{Aa}$ & $37 \mathrm{Aa}$ & $-(2)$ & $0,6 \mathrm{Bb}$ & $1,2 \mathrm{Ba}$ & $0,3 \mathrm{Cb}$ & $4,8 \mathrm{Aa}$ & $1,6 \mathrm{Ab}$ & $10 \mathrm{Bc}$ & $113 \mathrm{Aa}$ & $70 \mathrm{Ab}$ \\
\hline G. etunicatum (GE) & $40 \mathrm{Aa}$ & 69Aa & $40 \mathrm{Aa}$ & $0,7 \mathrm{Ab}$ & $1,4 \mathrm{Aa}$ & $1,4 \mathrm{ABa}$ & $3,7 \mathrm{Bab}$ & $4,0 \mathrm{Aa}$ & $2,3 \mathrm{Ab}$ & $82 \mathrm{Aab}$ & $93 \mathrm{ABa}$ & $61 \mathrm{Ab}$ \\
\hline \multirow[t]{2}{*}{$\mathrm{GE}+$ Mycoform } & $68 \mathrm{Aa}$ & $53 \mathrm{Aa}$ & $80 \mathrm{Aa}$ & $0,8 \mathrm{Ac}$ & $1,2 \mathrm{Ab}$ & $1,7 \mathrm{Aa}$ & $6,6 \mathrm{Aa}$ & $3,5 \mathrm{Ab}$ & $2,5 \mathrm{Ab}$ & $71 \mathrm{Aa}$ & $78 \mathrm{Ba}$ & $77 \mathrm{Aa}$ \\
\hline & \multicolumn{12}{|c|}{ Mutamba } \\
\hline Sem inoculação (SI) & - & $18 \mathrm{Aa}$ & $21 \mathrm{Aa}$ & - & $0,7 \mathrm{Bb}$ & $1,5 \mathrm{Aa}$ & - & $5,0 \mathrm{Aa}$ & $2,6 \mathrm{Ab}$ & & 196Aa & $147 \mathrm{Ab}$ \\
\hline G. etunicatum (GE) & $22 \mathrm{Aa}$ & $18 \mathrm{Aa}$ & $18 \mathrm{Aa}$ & $0,8 \mathrm{Ac}$ & $1,2 \mathrm{Ab}$ & $1,6 \mathrm{Aa}$ & $9,6 \mathrm{Aa}$ & $4,2 \mathrm{Ab}$ & $3,3 \mathrm{Ab}$ & $156 \mathrm{Aa}$ & $126 \mathrm{Ba}$ & $119 \mathrm{Aa}$ \\
\hline \multirow[t]{2}{*}{$\mathrm{GE}+$ Mycoform } & $21 \mathrm{Aa}$ & $17 \mathrm{Aa}$ & $17 \mathrm{Aa}$ & $0,9 \mathrm{Ac}$ & $1,3 \mathrm{Ab}$ & $1,7 \mathrm{Aa}$ & $9,7 \mathrm{Aa}$ & $5,5 \mathrm{Ab}$ & $3,3 \mathrm{Ac}$ & $144 \mathrm{Aa}$ & $118 \mathrm{Ba}$ & $111 \mathrm{Aa}$ \\
\hline & \multicolumn{12}{|c|}{ Cássia-verrugosa } \\
\hline Sem inoculação (SI) & - & $23 \mathrm{Aa}$ & $18 \mathrm{Aa}$ & - & $1,0 \mathrm{Bb}$ & $1,5 \mathrm{Ba}$ & - & $1,3 \mathrm{Aa}$ & $1,2 \mathrm{Aa}$ & - & $70 \mathrm{Aa}$ & $41 \mathrm{Ab}$ \\
\hline G. etunicatum (GE) & $20 \mathrm{Aa}$ & $19 \mathrm{Aa}$ & $18 \mathrm{Aa}$ & $0,8 \mathrm{Ac}$ & $1,5 \mathrm{Ab}$ & $1,8 \mathrm{Aa}$ & $3,3 \mathrm{Aa}$ & $0,5 \mathrm{Ab}$ & $0,5 \mathrm{Ab}$ & $44 \mathrm{Aa}$ & $42 \mathrm{Ba}$ & $36 \mathrm{Aa}$ \\
\hline \multirow[t]{2}{*}{$\mathrm{GE}+$ Mycoform } & $24 \mathrm{Aa}$ & $18 \mathrm{Aa}$ & $18 \mathrm{Aa}$ & $0,9 \mathrm{Ac}$ & $1,4 \mathrm{Ab}$ & $1,8 \mathrm{Aa}$ & $3,8 \mathrm{Aa}$ & $1,3 \mathrm{Ab}$ & $1,4 \mathrm{Ab}$ & $39 \mathrm{Aa}$ & $44 \mathrm{Ba}$ & $40 \mathrm{Ab}$ \\
\hline & \multicolumn{12}{|c|}{ Gravitinga } \\
\hline Sem inoculação (SI) & - & $24 \mathrm{Aa}$ & $14 \mathrm{Ab}$ & - & $1,0 \mathrm{Bb}$ & $1,5 \mathrm{Ba}$ & - & $4,0 \mathrm{Ba}$ & $3,0 \mathrm{Aa}$ & - & $133 \mathrm{Aa}$ & $77 \mathrm{Ab}$ \\
\hline G. etunicatum (GE) & 30Aa & $19 \mathrm{Bb}$ & $15 \mathrm{Ab}$ & $0,9 \mathrm{Ac}$ & $1,5 \mathrm{Ab}$ & $1,8 \mathrm{Aa}$ & $13,0 \mathrm{Aa}$ & $7,0 \mathrm{Ab}$ & $4,0 \mathrm{Ac}$ & $142 \mathrm{Aa}$ & $111 \mathrm{ABab}$ & $63 \mathrm{Ab}$ \\
\hline \multirow[t]{2}{*}{$\mathrm{GE}+$ Mycoform } & $29 \mathrm{Aa}$ & $15 \mathrm{Bb}$ & $16 \mathrm{Ab}$ & $1,1 \mathrm{Ab}$ & $1,3 \mathrm{Ab}$ & $1,9 \mathrm{Aa}$ & $10,0 \mathrm{Ba}$ & $6,0 \mathrm{ABb}$ & $3,0 \mathrm{Ac}$ & $90 \mathrm{Aa}$ & $66 \mathrm{Ba}$ & $58 \mathrm{Aa}$ \\
\hline & & & & & & & Aroeira & & & & & \\
\hline Sem inoculação (SI) & 30Aa & $22 \mathrm{Ab}$ & $13 \mathrm{Ac}$ & - & - & $1,4 \mathrm{Aa}$ & $0,2 \mathrm{Bb}$ & $0,6 \mathrm{Bb}$ & $2,1 \mathrm{Bb}$ & $8 \mathrm{Bb}$ & $5 \mathrm{Cb}$ & $60 \mathrm{Aa}$ \\
\hline G. etunicatum (GE) & $22 \mathrm{Ba}$ & $17 \mathrm{Ba}$ & $12 \mathrm{Ab}$ & $1,0 \mathrm{Ab}$ & $1,7 \mathrm{Aa}$ & $1,5 \mathrm{Aa}$ & $6,8 \mathrm{Aa}$ & $6,2 \mathrm{Aa}$ & $3,3 \mathrm{Ab}$ & $68 \mathrm{Aa}$ & $72 \mathrm{Aa}$ & $56 \mathrm{Aa}$ \\
\hline $\mathrm{GE}+$ Mycoform & $20 \mathrm{Ba}$ & $12 \mathrm{Cb}$ & $16 \mathrm{Aab}$ & $1,0 \mathrm{Ab}$ & $1,5 \mathrm{Aa}$ & $1,6 \mathrm{Aa}$ & $7,3 \mathrm{Aa}$ & $5,7 \mathrm{Ab}$ & $3,0 \mathrm{ABc}$ & $61 \mathrm{Aa}$ & $49 \mathrm{Ba}$ & $61 \mathrm{Aa}$ \\
\hline & \multicolumn{12}{|c|}{ Trema } \\
\hline Sem inoculação (SI) & - & $24 \mathrm{Aa}$ & $15 \mathrm{Ab}$ & - & $1,1 \mathrm{Ab}$ & $2,2 \mathrm{Aa}$ & - & $4,2 \mathrm{Aa}$ & $2,7 \mathrm{Bb}$ & - & $198 \mathrm{Aa}$ & $123 \mathrm{Bb}$ \\
\hline G. etunicatum (GE) & $23 \mathrm{Aa}$ & $16 \mathrm{Bb}$ & $18 \mathrm{Ab}$ & $0,7 \mathrm{Ac}$ & $1,1 \mathrm{Ab}$ & $2,3 \mathrm{Aa}$ & $4,2 \mathrm{Ba}$ & $3,5 \mathrm{Aa}$ & $4,1 \mathrm{Aa}$ & $136 \mathrm{Bab}$ & $110 \mathrm{Bb}$ & 159Aa \\
\hline $\mathrm{GE}+$ Mycoform & $24 \mathrm{Aa}$ & $14 \mathrm{Bb}$ & $15 \mathrm{Ab}$ & $0,9 \mathrm{Ab}$ & $1,1 \mathrm{Ab}$ & $2,0 \mathrm{Aa}$ & $5,6 \mathrm{Aa}$ & $3,2 \mathrm{Ab}$ & $2,9 \mathrm{Bb}$ & $181 \mathrm{Aa}$ & $117 \mathrm{Bb}$ & $128 \mathrm{ABb}$ \\
\hline
\end{tabular}

${ }^{(1)}$ Médias seguidas da mesma letra, maiúscula na coluna e minúscula na linha, dentro de cada espécie e para cada nutriente, não diferem entre si pelo teste de Tukey a $5 \%$ de probabilidade. ${ }^{(2)}$ Material insuficiente para análise. 
solo. Isto sugere que a formononetina possa ter algum papel no controle da colonização e não apenas estimular a colonização.

A dominância do fedegoso e da cássia-verrugosa nas bandejas sem inóculo e com $\mathrm{P}$ muito baixo $\left(0,002 \mathrm{mg} \mathrm{L}^{-1}\right)$ deve-se mais à incapacidade das demais espécies de crescerem nesta condição de $\mathrm{P}$ sem micorrizas do que à competição por luz, espaço ou nutrientes entre as plantas. Tanto que, neste nível de P no solo, ao receberem inoculação, as espécies apresentaram crescimento mais equilibrado (Figura 3). Ao contrário, a dominância da gravitinga em relação às demais espécies nos níveis 0,02 e $0,2 \mathrm{mg} \mathrm{L}^{-1}$ de $\mathrm{P}$ evidencia a maior capacidade desta espécie em competir pelo P disponível no solo, o que a torna

Tabela 2. Colonização micorrízica (\%) das raízes de espécies arbóreas nativas aos $120 \mathrm{DAS}$, em solo com 0,002 , 0,02 e $0,2 \mathrm{mg} \mathrm{L}^{-1}$ de fósforo ${ }^{(1)}$.

\begin{tabular}{|c|c|c|c|}
\hline \multirow{2}{*}{$\begin{array}{l}\text { Tratamento de } \\
\text { inoculação }\end{array}$} & \multicolumn{3}{|c|}{ Doses de fósforo } \\
\hline & 0,002 & 0,02 & 0,2 \\
\hline & \multicolumn{3}{|c|}{ Fedegoso } \\
\hline Sem inoculação (SI) & $0 \mathrm{Ba}$ & $0 \mathrm{Ba}$ & $0 \mathrm{Ba}$ \\
\hline G. etunicatum (GE) & $44 \mathrm{Aa}$ & $43 \mathrm{Aa}$ & $43 \mathrm{Aa}$ \\
\hline $\mathrm{GE}+$ Mycoform & $57 \mathrm{Aa}$ & $57 \mathrm{Aa}$ & $38 \mathrm{Aa}$ \\
\hline & \multicolumn{3}{|c|}{ Mutamba } \\
\hline Sem inoculação (SI) & $0 \mathrm{Ca}$ & $0 \mathrm{Ba}$ & $0 \mathrm{Ba}$ \\
\hline G. etunicatum (GE) & $15 \mathrm{Ba}$ & $28 \mathrm{Aa}$ & $23 \mathrm{Aa}$ \\
\hline \multirow[t]{2}{*}{ GE + Mycoform } & $62 \mathrm{Aa}$ & $31 \mathrm{Ab}$ & $26 \mathrm{Ab}$ \\
\hline & \multicolumn{3}{|c|}{ Cássia-verrugosa } \\
\hline Sem inoculação (SI) & $0 \mathrm{Ba}$ & $0 \mathrm{Ba}$ & $0 \mathrm{Ba}$ \\
\hline G. etunicatum (GE) & $34 \mathrm{Aa}$ & $45 \mathrm{Aa}$ & $17 \mathrm{Aa}$ \\
\hline $\mathrm{GE}+$ Mycoform & $26 \mathrm{Aa}$ & $23 \mathrm{Aa}$ & $25 \mathrm{Aa}$ \\
\hline & \multicolumn{3}{|c|}{ Gravitinga } \\
\hline Sem inoculação (SI) & $0 \mathrm{Ca}$ & $0 \mathrm{Ca}$ & $0 \mathrm{Ca}$ \\
\hline G. etunicatum (GE) & $15 \mathrm{Ba}$ & $11 \mathrm{Aa}$ & $15 \mathrm{Aa}$ \\
\hline $\mathrm{GE}+$ Mycoform & $32 \mathrm{Aa}$ & $5 \mathrm{Bb}$ & $5 \mathrm{Bb}$ \\
\hline & \multicolumn{3}{|c|}{ Aroeira } \\
\hline Sem inoculação (SI) & $0 \mathrm{Ba}$ & $0 \mathrm{Bb}$ & $0 \mathrm{Ba}$ \\
\hline G. etunicatum (GE) & $44 \mathrm{Aa}$ & $46 \mathrm{Aa}$ & $33 \mathrm{Aa}$ \\
\hline $\mathrm{GE}+$ Mycoform & $45 \mathrm{Aa}$ & $63 \mathrm{Aa}$ & $43 \mathrm{Aa}$ \\
\hline & \multicolumn{3}{|c|}{ Trema } \\
\hline Sem inoculação (SI) & $\mathrm{OBa}$ & $0 \mathrm{Ca}$ & $0 \mathrm{Ba}$ \\
\hline G. etunicatum (GE) & 38Aa & $34 \mathrm{Aa}$ & $24 \mathrm{Aa}$ \\
\hline $\mathrm{GE}+$ Mycoform & $34 \mathrm{Aa}$ & $13 \mathrm{Bb}$ & $28 \mathrm{Aa}$ \\
\hline
\end{tabular}

${ }^{(1)}$ Médias seguidas de mesma letra, maiúscula na coluna e minúscula na linha, dentro de cada espécie, não diferem entre si pelo teste de Tukey a 5\% de probabilidade; Mycoform: formononetina (4'metoxi 7-hidroxiisoflavona). também mais competitiva por espaço e luz. A micorrização das plantas teve dois papéis contrários, dependendo do $\mathrm{P}$ disponível no solo. Assim, no nível 0,02 $\mathrm{mg} \mathrm{L}^{-1}$ de $\mathrm{P}$ a inoculação de GE diminuiu a dominância da gravitinga e favoreceu o crescimento das demais espécies, possivelmente por tornarem-nas mais competitivas por nutrientes, particularmente P, e espaço. Portanto, a micorrização das plantas promove maior uniformidade e crescimento mais balanceado entre as espécies. Isto, em condições naturais, favoreceria a diversidade vegetal em plantios mistos de espécies florestais e, portanto, seria de grande interesse no reflorestamento. Ao contrário, em condições de alto $\mathrm{P}\left(0,2 \mathrm{mg} \mathrm{L}^{-1}\right)$, em que a micorrização foi pouco efetiva em promover o crescimento das plantas, chegando até mesmo a prejudicálo, como ocorreu na aroeira e na trema, a inoculação favoreceu a dominância da gravitinga. Tal efeito sugere que a micorriza tem papel importante na dinâmica e na interação entre membros das comunidades vegetais pela influência na competição e na interferência na sucessão vegetal, conforme já sugerido por Janos (1987). Em área reflorestada por semeadura direta isto teria grande importância, pois a diversidade vegetal, essencial ao sucesso desses programas, seria afetada pela disponibilidade de P no solo. Em solos de baixa fertilidade, a presença abundante de propágulos de FMAs ou a introdução destes torna-se de grande interesse prático, para atingir plantios com diversidade vegetal e crescimento equilibrado entre as espécies. Assim, estudos sobre a peletização de sementes de espécies nativas com fosfatos e fungos micorrízicos seriam de grande interesse.

\section{Conclusões}

1. O crescimento inicial das espécies arbóreas pioneiras em semeadura mista e direta é favorecido pela elevação da disponibilidade de P no solo e pela inoculação de Glomus etunicatum.

2. Glomus etunicatum só beneficia o desenvolvimento das plantas em condições de baixo P disponível no solo.

3. A aplicação de Mycoform afeta pouco o crescimento e a nutrição das plantas e a competição entre elas. 
4. O fedegoso cresce em solo com extrema deficiência de $\mathrm{P}$, particularmente se micorrizado.

5. Com suprimento adequado de $\mathrm{P}$, a gravitinga é competitiva e mostra elevada dominância em semeadura direta mista.

6. A micorrização influencia as relações competitivas entre as espécies, podendo tanto diminuir quanto aumentar a competição de espécies em semeadura simultânea dependendo da disponibilidade de P no solo.

\section{Referências}

BRANDON, N. J.; SHELTON, H. M.; PECK, D. M. Factors affecting the early growth of Leucaena leucocephala - 2: importance of arbuscular mycorrhizal fungi, grass competition and phosphorus application on yield and nodulation of leucaena in pots. Australian Journal of Experimental Agriculture, Melbourne, v. 37 , n. 1, p. 35-43, Jan. 1997.

CARNEIRO, M. A. C.; SIQUEIRA, J. O.; DAVIDE, A. C.; GOMES, L. J.; CURI, N.; VALE, F. R. do. Fungo micorrízico e superfosfato no crescimento de espécies arbóreas tropicais. Scientia Forestalis, Piracicaba, n. 50, p. 21-36, dez. 1996.

DAVIDE, A. C.; FARIA, J. M. R.; BOTELHO, S. A. Propagação de espécies florestais. Belo Horizonte: Cemig/ Ufla, 1995. 41 p.

FAO (Roma, Itália). State of the world's forests, 1997. Rome, 1997. 200 p.

GIOVANNETTI, M.; MOSSE, B. An evaluation of techniques to measure vesicular-arbuscular mycorrhizal infection in roots. New Phytologist, Oxford, v. 84, n. 3, p. 484-500, Mar. 1980.

HABTE, M.; MANJUnATH, A. Categories of vesicular-arbuscular mycorrhizal dependency of host species. Mycorrhiza, Heidelberg, v. 1, n. 1, p. 3-12, Sept. 1991.

JANOS, D. P. VA mycorrhizas in humid tropical ecosystems. In: SAFIR, G. R. (Ed.). Ecophysiology of VA mycorrhizal plants. Boca Raton: CRC Press, 1987. p. 107-134.

KNIGHT, A. J. P.; BEALE, P. E.; DALTON, G. S. Direct seeding of native trees and shrubs in low rainfall areas and on non-wetting sands in South Australia. Agroforestry Systems, The Hague, v. 39, n. 3, p. 225-239, Jan. 1998.
LIMA, H. N.; VALE, F. R. do; SIQUEIRA, J. O.; CURI, N. Crescimento inicial a campo de sete espécies arbóreas nativas em resposta à adubação mineral com NPK. Ciência e Agrotecnologia, Lavras, v. 21, n. 2, p. 225-239, abr. 1997.

MANJUNATH, A.; HABTE, M. Establishment of soil solution P levels for studies involving vesicular-arbuscular mycorrhizal symbiosis. Communications in Soil Science and Plant Analysis, New York, v. 21, n. 7/8, p. 557-566, May 1990.

MATTEI, V. L. Semeadura direta: uma alternativa para implantação de povoamentos de Pinus taeda L. In: CONGRESSO FLORESTAL PANAMERICANO, 1.; CONGRESSO FLORESTAL BRASILEIRO, 7., 1993, Curitiba. Anais... São Paulo: Sociedade Brasileira de Silvicultura, 1993. v. 1, p. 306-308.

MONTAGNINI, F.; SANCHO, F. Nutrient budgets of young plantations with native trees: strategies for sustained management. In: BENTLEY, W.; GOWEN, M. (Ed.). Forest resources and wood-based biomass energy as rural development assets. New Delhi: IBH Publishing, 1994. p. 213-233.

PARON, M. E.; SIQUEIRA, J. O.; CURI, N. Fungo micorrízico, fósforo e nitrogênio no crescimento inicial da trema e do fedegoso. Revista Brasileira de Ciência do Solo, Viçosa, MG, v. 21, n. 4, p. 567-574, out./dez. 1997.

PHILLIPS, J. M.; HAYMAN, D. S. Improved procedures for clearing roots and staining parasitic and vesicular-arbuscular mycorrhizal fungi for rapid assessment of infection. Transactions of the British Mycological Society, London, v. 55, p. 158-161, 1970.

POMPÉIA, S. L.; PRADELLA, D. Z. A.; MARTINS, S. E.; SANTOS, R. C.; DINIZ, K. M. A semeadura aérea na Serra do Mar em Cubatão. Revista Ambiente, São Paulo, v. 3, n. 1, p. 13-19, 1989.

POUYÚ-ROJAS, E.; SIQUEIRA, J. O. Micorriza arbuscular e fertilização do solo no desenvolvimento pós-transplante de mudas de sete espécies florestais. Pesquisa Agropecuária Brasileira, Brasília, v. 35, n. 1, p. 103-114, jan. 2000.

SAGGIN JÚNIOR, O. J. Micorrizas arbusculares em mudas de espécies arbóreas do Sudeste brasileiro. 1997. 120 f. Tese (Doutorado em Solos e Nutrição de Plantas) - Universidade Federal de Lavras, Lavras, 1997.

SAGGIN JÚNIOR, O. J.; LOVATO, P. E. Aplicação de micorrizas arbusculares na produção de mudas e plantas 
micropropagadas. In: SIQUEIRA, J. O.; MOREIRA, F. M. S.; LOPES, A. S.; GUILHERME, L. R. G.; FAQUIN, V.; FURTINI NETO, A. E.; CARVALHO, J. G. (Ed.). Inter-relação fertilidade, biologia do solo e nutrição de plantas. Viçosa, MG: Sociedade Brasileira de Ciência do Solo/Ufla, 1999. p. 725-774.

SILVA JÚNIOR, J. P. da; SIQUEIRA, J. O. Colonização micorrízica e crescimento da soja com diferentes fungos e aplicação do isoflavonóide formononetina. Pesquisa Agropecuária Brasileira, Brasília, v. 33, n. 6, p. 953-959, jun. 1998.

SIQUEIRA, J. O.; SAGGIN JÚNIOR, O. J. Dependency on arbuscular mycorrhizal fungi and responsiveness of some Brazilian native woody species. Mycorrhiza, Heidelberg, v. 11, n. 5, p. 245-255, Oct. 2001.

SIQUEIRA, J. O.; SAGGIN JÚNIOR, O. J. The importance of mycorrhizae association in natural in low fertility. In: INTERNATIONAL SYMPOSIUM ON ENVIRONMENTAL STRESS, 1992, Belo Horizonte.
Proceedings... Sete Lagoas: Embrapa-CNPMS, 1995. p. $240-280$.

SIQUEIRA, J. O.; CARNEIRO, M. A. C.; CURI, N.; ROSADO, S. C. S.; DAVIDE, A. C. Mycorrhizal colonization and mycotrophic growth of native woody species as related to successional groups in southeastern Brazil. Forest Ecology and Management, Amsterdam, v. 107, n. 1/3, p. 241-252, Aug. 1998.

SIQUEIRA, J. O.; SAFIR, G. R.; NAIR, M. G. Stimulation of vesicular-arbuscular mycorrhiza formation and growth of white clover by flavonoid compounds. New Phytologist, Oxford, v. 118, n. 1, p. 87-93, May 1991

SMITH, S. E.; ROBSON, A. D.; ABBOTT, L. K. The involvement of mycorrhizas in assessment of genetically dependent efficiency of nutrient uptake and use. Plant and Soil, Dordrecht, v. 146, n. 1/2, p. 169-179, 1992.

SOUZA, F. A. de; SILVA, E. M. R. da. Micorrizas arbusculares na revegetação de áreas degradadas. In: SIQUEIRA, J. O. (Ed.). Avanços em fundamentos e aplicação de micorrizas. Lavras: Ufla, 1996. p. 255-290. 